\title{
C9ORF72 dipeptide repeat proteins disrupt formation of GEM bodies and induce aberrant accumulation of survival of motor neuron protein
}

Yuma Kato ${ }^{1, *}$, Minnie Yokogawa ${ }^{1, *}$, Ikuma Nakagawa ${ }^{1}$, Kazunari Onodera ${ }^{2}$, Hideyuki Okano $^{3}$, Haruhisa Inoue ${ }^{4,5,6}$, Mitsuharu Hattori ${ }^{1}$, Yohei Okada ${ }^{2, \#}$, and Hitomi Tsuiji ${ }^{1, \#}$

${ }^{1}$ Department of Biomedical Science, Graduate School of Pharmaceutical Sciences, Nagoya City University, Nagoya, Aichi 467-8603, Japan

${ }^{2}$ Department of Neurology, Aichi Medical University School of Medicine, Nagakute, Aichi 480-1195, Japan

${ }^{3}$ Department of Physiology, Keio University School of Medicine, Shinjuku, Tokyo 1608582, Japan

${ }^{4}$ Center for iPS Cell Research and Application, Kyoto University, Kyoto 606-8507, Japan

5iPSC-Based Drug Discovery and Development Team, RIKEN BioResource Research Center (BRC), Kyoto 619-0237, Japan

${ }^{6}$ Medical-risk Avoidance based on iPS Cells Team, RIKEN Center for Advanced Intelligence Project (AIP), Kyoto 606-8507, Japan

* Contributed equally

\# To whom correspondence should be addressed:

Hitomi Tsuiji, Ph.D.

3-1 Tanabe-dori, Mizuho-ku, Nagoya, Aichi 467-8603, Japan

TEL: +81-52-836-3789

E-mail: hitomitsuiji@phar.nagoya-cu.ac.jp

Yohei Okada, M.D., Ph.D.

1-1 Yazakokarimata, Nagakute, Aichi 480-1195, Japan

TEL: +81-561-62-3311 


\title{
E-mail: yohei@aichi-med-u.ac.jp
}

Keywords

ALS, C9ORF72, SMN, iPSCs, dipeptide repeat proteins

\begin{abstract}
A GGGGCC repeat expansion in the C9ORF72 gene is the most common genetic cause of amyotrophic lateral sclerosis (ALS), a devastating motor neuron disease. In the neurons of ALS patients, dipeptide repeat proteins (DPRs) are produced from repeat-containing RNAs by an unconventional form of translation, and some of these proteins, especially those containing poly(glycine-arginine) and poly(proline-arginine), are toxic to neurons. Gemini of coiled bodies (GEMs) are nuclear structures that harbor survival of motor neuron (SMN) protein, and SMN is essential for the assembly of Urich small nuclear ribonucleoproteins (snRNPs) that are central for splicing. We previously reported that GEMs are lost and that snRNP biogenesis is misregulated in the motor neurons of ALS patients. Here we show that DPRs interfere with GEM formation and proper SMN localization in HeLa cells and iPSC-derived motor neurons from an ALS patient with the C9ORF72 mutation. The accumulation of poly(glycinearginine) markedly reduced the number of GEMs and caused the formation of aberrant cytoplasmic RNA granules that sequestered SMN. These findings indicate the functional impairment of SMN in motor neurons expressing DPRs and may provide a mechanism to explain the vulnerability of motor neurons of C9ORF72-ALS patients.
\end{abstract}




\section{Introduction}

Amyotrophic lateral sclerosis (ALS) is a late-onset devastating motor neuron disease, characterized by progressive loss of motor neurons (MNs) resulting in muscle weakness and atrophy ${ }^{1}$. A large expansion of a GGGGCC nucleotide repeat in the first intron of the C9ORF72 gene is the most common genetic cause of ALS and frontotemporal dementia (FTD) (C9-ALS/FTD) ${ }^{2,3}$. Both sense and antisense strands of the expanded repeat are transcribed, and the resulting RNAs are translated by an unconventional form of translation called repeat-associated non-AUG (RAN) translation to produce dipeptide repeat (DPR) proteins ${ }^{4-6}$. These DPR proteins accumulate in the affected neurons of patients with C9ORF72 mutations and represent a pathological hallmark. DPRs are cytotoxic in cells and multiple animal models ${ }^{7-10}$. Among them, the arginine-rich ones poly(glycine-arginine, GR) and poly(proline-arginine, PR) are the most toxic to neurons ${ }^{7-9}$. The pattern of $\operatorname{poly}(G R)$ accumulation seems to be the most correlated with neurodegeneration ${ }^{11,12}$.

On the other hand, spinal muscular atrophy (SMA) is early-onset motor neuron disease, caused by the loss of survival of motor neuron (SMN) protein, due to mutation in the SMN1 gene ${ }^{13-16}$. SMN is essential for the biogenesis of U-rich small nuclear ribonucleoproteins (snRNPs), which are the major components of the spliceosome, the machinery that carries out pre-mRNA splicing ${ }^{17}$. Accordingly, there is a tight correlation between the degree of SMN deficiency and snRNP assembly in SMA ${ }^{17,18}$. In vertebrates, GEMs (Gemini of coiled bodies) consist of SMN, seven Gemin proteins (Gemin 2-Gemin 8), and Unrip. The precise function of GEMs is still unclear, although the amount of SMN protein correlates well with the number of GEMs, and the number of GEMs is considered to be a good indicator of SMA severity ${ }^{19}$. snRNPs consist of one or two small nuclear RNAs (snRNAs) bound to a set of seven Smith (Sm) or Smithlike proteins and a unique set of snRNP-specific proteins ${ }^{20}$. U1, U2, U4/U6, and U5 snRNPs form the major spliceosome, which is responsible for splicing of a majority of pre-mRNA introns ${ }^{21}$. In SMA patients, SMN deficiency causes abnormal snRNP 
biogenesis and widespread defects in splicing that might be the major causes of neurodegeneration ${ }^{18}$.

We and others have previously found that GEMs, the site of SMN protein localization in the nucleus, are lost and that snRNP biogenesis is dysregulated in affected MNs of patients with ALS and SMA ${ }^{22,23}$. Loss of GEMs and/or abnormal snRNP biogenesis has also been observed in fibroblasts of ALS patients harboring TDP-43 or FUS mutations ${ }^{24}$ and in various ALS mouse models expressing mutant SOD1 or TDP-43 ${ }^{25,26}$. Enhancing the level of SMN protein attenuates neurodegeneration in a mouse model of SMA, transgenic mutant TDP-43 mice ${ }^{27}$ and mutant SOD1 mice ${ }^{28}$. SMN directly binds to FUS, another RNA-binding protein associated with ALS, providing further evidence for the link between ALS and SMA ${ }^{22,24,29}$. These findings underscore the importance of SMN protein and proper snRNP biogenesis in ALS pathogenesis. However, the direct causes of loss of GEMs and abnormal snRNP biogenesis in ALS patients have been obscure.

In this study, we show that poly(GR) and poly(PR) affect the localization of SMN and result in the loss of GEMs and the cytoplasmic aggregation of SMN and RNAbinding proteins. We also provide evidence for decreased number of GEMs in MNs differentiated from iPSCs of an ALS patient with a C9ORF72 mutation.

\section{Results}

\section{Poly(GR) disrupts GEM formation}

We first investigated the effect of C9ORF72 poly(GR) and poly(PR) on GEM formation. We transfected HeLa cells with a plasmid encoding a FLAG-tagged construct with 50 repeats of glycine-arginine (poly(GR)) or a FLAG-tagged construct with 50 repeats of proline-arginine (poly(PR)). We detected GEMs by immunostaining for SMN protein and detected DPRs using an anti-FLAG antibody. Both poly(GR) and poly(PR) 
localized to the nucleolus (Fig. 1a), consistent with previous reports ${ }^{7}$, whereas poly(GR) also distributed in the cytoplasm very weakly (data not shown). In HeLa cells transfected with an empty vector, we observed approximately 8 GEMs per cell (Fig. 1a, upper row, 1B). In cells with a poly(GR) accumulation in the nucleolus, the number of GEMs was reduced to approximately 2 (Fig. 1a, middle row,1b). Poly(PR) accumulation in the nucleolus considerably reduced the number of GEMs, but to a lesser extent than poly(GR) and not in a statistically significant way (Fig. 1a, bottom row, $1 b, p=0.0768)$. These observations clearly indicate that DPRs especially poly(GR) disrupts the formation of GEMs in nucleus.

\section{Poly(GR) and poly(PR) induce cytoplasmic RNA granules harboring SMN protein}

We also noticed that SMN and poly(GR) occasionally accumulated in the cytoplasm of cells expressing poly(GR). Therefore, we next investigated whether DPRs induce abnormal cytoplasmic granules. Poly(A)-binding protein (PABP) was distributed diffusely in the cytoplasm of cells with mock transfection, whereas PABP formed foci resembling stress granules in the cytoplasm of cells expressing poly(GR) (Fig. 2a). We observed cytoplasmic accumulation of PABP in approximately $80 \%$ of cells with poly(GR) expression in the nucleolus (Fig. 2b). Some PABP accumulations harbored poly(GR) (Fig. 2a), and SMN occasionally colocalized with poly(GR) or PABP in the cytoplasm (Fig. 2c, 2d) . These PABP- and poly(GR)-containing cytoplasmic granules may also contain RNA, which we visualized by SYTO RNASelect Green Fluorescent Cell Stain (Fig. 2e). Poly(PR) also led to the formation of PABP granules resembling stress granules but to a lesser extent than poly(GR) (Fig. 2a, bottom row, 2b, p = 0.0158). Notably, poly(PR) did not colocalize with PABP in cytoplasmic granules induced by poly(PR) (Fig. 2a, bottom row), though SMN occasionally localized in the cytoplasmic PABP granules (Fig. 2b, 2c). Intriguingly, the average number of GEMs was reduced to 1.23 in the cells with SMN accumulation in the cytoplasm $(n=3$, more 
than 10 cells were counted). Together, these findings suggest that C9ORF72 DPRs can disrupt GEMs and cause mislocalization of SMN protein to the cytoplasm, where they accumulate with RNA protein granules containing PABP.

\section{Poly(PR) expression combined with oxidative stress induces persistent cytoplasmic stress granules harboring SMN}

Based on the results of these experiments in which poly $(\mathrm{GR})$ and poly $(\mathrm{PR})$ caused the formation of cytoplasmic PABP granules resembling stress granules, and SMN was inactivated by oxidative stress ${ }^{30}$, we next tested whether stress granule formation could induce loss of GEMs. HeLa cells were treated with $500 \mu \mathrm{M}$ sodium arsenite for 30 min and immunostained with anti-PABP and anti-SMN antibodies. The formation of stress granules itself did not induce the loss of GEMs (Supplementary Fig. S1), indicating that sequestration of SMN in stress granules is not sufficient to induce the loss of GEMs. We further tested the combined effects of DPR toxicity and oxidative stress on SMN. We treated HeLa cells with $500 \mu \mathrm{M}$ sodium arsenite for 30 min to induce oxidative stress and PABP-containing stress granule formation. Following arsenite treatment, we washed the cells to remove the arsenite and allowed the cells to recover and the stress granules to disassemble. In control cells, the stress granules disassembled almost completely, and PABP staining was dispersed 3 hours postincubation. However, in cells expressing poly(PR), cytoplasmic granules became more persistent and remained in the cytoplasm even after 6 hours (Fig. 3a, 3b). This is consistent with other reports showing that DPRs can induce persistent stress granule formation ${ }^{31,32}$. These persistent cytoplasmic granules occasionally harbored SMN protein (Fig. 3b), and the average number of GEMs was reduced to 0.7 in these cells $(n=3$, more than 10 cells were counted). This sequestration of SMN in abnormal cytoplasmic granules could disturb the normal function of SMN (illustrated in Fig. 5).

\section{The number of GEMs in MNs derived from iPSCs of a C9ORF72-ALS patient is reduced}


We previously showed that GEMs are almost completely lost in MNs in the spinal cord of postmortem ALS patients ${ }^{22}$. To provide insight whether C9ORF72 mutation affect GEM pathology in patient-derived MNs, we took advantage of human iPSCs derived from an ALS patient with a C9ORF72 mutation (C9-ALS) and two healthy controls. The origins of the iPSC clones used in this analysis are described in Table 1. Differentiation of iPSCs into MNs was performed as previously described ${ }^{33-35}$ (Fig. 4a). The differentiation efficiency into MNs was approximately the same in all three iPSCs used in this study, as evidenced by HB9 and Isl-1 immunostaining at day 7 of adherent differentiation (Fig. 4b). MNs differentiated from iPSCs derived from an ALS patient with a C9ORF72 mutation (C9-ALS) or control patients were fixed for the analysis at 4 weeks of adherent differentiation. iPSC-derived MNs were visualized by the lentivirus reporter specific for motor neurons (HB9 ${ }^{\mathrm{e} 338}:$ :Venus). To count the number of GEMs, we immunostained MNs with anti-SMN and anti-Gemin-2 antibodies, and SMN-positive and Gemin-2-positive granules in the nuclei were counted as GEMs. We observed a decreased number of GEMs in MNs differentiated from iPSCs of the ALS patient compared with that in MNs from healthy donors (Fig. 4c, $p=0.0002$ (Contol1 vs C9ALS) and $=0.0002$ (Contol2 vs C9-ALS), 4d).

We have also previously shown that snRNPs extensively accumulate in MNs of postmortem tissues of ALS patients ${ }^{22}$. To investigate whether snRNP accumulation occurred in MNs differentiated from the C9-ALS patient, we immunostained MNs with anti-Sm and anti-U1 70K antibodies. Anti-Sm antibody $\mathrm{Y} 12$ recognizes the methylated Sm core proteins of most snRNPs. U1 70K is a U1 snRNP-specific protein. There was no significant accumulation nor changes in their distribution (Supplementary Fig. S2), indicating that snRNP accumulation observed in the postmortem MNs of ALS patients seems to be the result of neurodegeneration. Together, these findings provide evidence for disruptions of GEMs in ALS patient-derived MNs harboring the C9ORF72 mutation, suggesting low levels of SMN in the MNs of ALS patients and the actual involvement of GEM pathology in the degeneration of MNs in C9-ALS patients. 


\section{Discussion}

In this study, we showed that the expression of the C9ORF72 DPR poly(GR) and, to a lesser extent, poly(PR) in HeLa cells results in the loss of GEM bodies. The analysis of MNs differentiated from iPSCs from an ALS patient harboring a C9ORF72 mutation showed a decreased number of GEMs in MNs. Since the number of GEMs is an indicator of the abundance of functional SMN ${ }^{19}$, these results indicate low levels of SMN in MNs of ALS patients harboring C9ORF72 mutations. Furthermore, poly(GR) and poly(PR) induced the abnormal accumulation of SMN in the cytoplasm, especially after oxidative stress. Since SMN has a crucial role in snRNP assembly, low levels of SMN and abnormal cytoplasmic accumulation of SMN could lead to disturbed snRNP biogenesis. Taken together, we provide evidence that the level of functional SMN is low in MNs harboring the C9ORF72 mutation.

What is the molecular mechanism to explain the loss of GEMs induced by poly(GR) or poly $(\mathrm{PR})$ ? Poly $(\mathrm{GR})$ and poly $(\mathrm{PR})$ have both been shown to interact with RNAbinding proteins and proteins with low complexity domains (also known as prion-like domains), thus damaging the assembly, dynamics, and functions of membrane-less phase-separated organelles ${ }^{31,32}$. Since GEMs are a type of membrane-less organelle in the nucleus, it is likely that poly $(G R)$ and poly(PR) directly alter the biophysical properties of GEMs by inhibiting liquid-liquid phase separation. GEMs often associate or overlap with Cajal bodies, where snRNAs undergo maturation, and some studies have shown that poly(GR) and poly(PR) impact the formation of Cajal bodies ${ }^{31,32}$. The sequestration of SMN in cytoplasmic PABP granules may reduce the amount of SMN in the nucleus, resulting in an acceleration of a loss of GEMs. Alternatively, DPRs cause defects in nucleocytoplasmic transport ${ }^{36-39}$, thus potentially leading to the retention of SMN in the cytoplasm. Other possible mechanisms leading to a low level of SMN are 
heterochromatin anomalies, dsRNA accumulation, and nucleolar stress induced by DPRs ${ }^{40,41}$. These stresses might induce the degradation of SMN.

In this study, the sequestration of SMN in cytoplasmic PABP granules was observed in cells with poly(GR) or poly(PR). Poly(GR), poly(PR), and SMN are all able to bind to $\mathrm{RNA}^{7}$, so perhaps they interact in an RNA-dependent manner. Alternatively, several proteins that interact with poly $(G R)$ or poly $(P R)$ have been reported ${ }^{31,42-44}$, and some of these might mediate the interaction with SMN and DPRs. Poly(GR) can bind ribosomal subunits and induce translational arrest ${ }^{40}$, and SMN has also been found to associate with the ribosome and have a role in translation ${ }^{45,46}$, indicating a possible link between DPRs and SMN.

C9ORF72 mutations can cause disease by several different mechanisms. They can induce a loss of the C9ORF72 protein, a toxic gain of function from C9ORF72 RNA accumulation or a toxic gain of function from the DPRs ${ }^{10}$. Among these, poly(GR) and poly $(P R)$ seem to be the most toxic to neurons ${ }^{7-9}$. The pattern of poly(GR) accumulation seems to be the most correlated with neurodegeneration ${ }^{11,12}$. Here, we showed that poly(GR) has stronger effects on GEM formation than poly(PR), that may explain the neurotoxicity of poly(GR) in ALS patients.

Here, we observed loss of GEMs but not abnormal snRNP accumulation in MNs differentiated from iPSCs of a C9ORF72-ALS patient at 4 weeks of differentiation. These observations may suggest that the loss of GEMs occurs at a relatively early stage and causes neurodegeneration and that snRNP accumulation is the result of the loss of SMN following neurodegeneration. The association of DPRs and U1 or U2 snRNP components ${ }^{31,42,44}$ might contribute to the abnormal snRNPs observed in ALS patients.

In this report, we provided evidence that the level of functional SMN is low in MNs harboring a C9ORF72 mutation. Importantly, increasing the level of SMN improves the survival of many types of human MNs, including MNs differentiated from iPSCs of SMA patients and ALS patients harboring TDP-43 mutations, even with mutations in the 
ALS-related SOD1 gene ${ }^{47}$. These findings indicate the beneficial effect of increasing functional SMN in MNs and now suggest that this type of approach could be explored for C9ORF72 mutations.

\section{Methods}

\section{Cell culture and transfection}

HeLa cells were maintained in DMEM/Ham's F-12 with L-glutamine medium (Thermo Fisher Scientific, USA) supplemented with $10 \%$ foetal bovine serum and a $0.5 \%$ penicillin-streptomycin mixed solution. In this study, a HeLa cell clone with a relatively large nucleus (HeLa-C3) was expanded and used. For the overexpression of DPRs, cells were transfected with plasmids using polyethylenimine "Max" (Polysciences, USA) with a standard method.

\section{iPSC culture and differentiation}

iPSCs were maintained on mitomycin-C-treated SNL murine fibroblast feeder cells in $0.1 \%$ gelatine-coated tissue culture dishes in hESC medium. iPSCs were differentiated into spinal MNs as previously described ${ }^{33-35}$. Briefly, iPSC colonies were detached using a dissociation solution (0.25\% trypsin, $100 \mu \mathrm{g} / \mathrm{ml}$ collagenase IV (Thermo Fisher Scientific, USA), $1 \mathrm{mM} \mathrm{CaCl}_{2}$, and 20\% KnockOut $^{\mathrm{TM}}$ Serum Replacement (KSR, Thermo Fisher Scientific, USA)) and cultured in suspension in bacteriological dishes in standard hESC medium. After the removal from SNL feeder cells, the cells were incubated for 1-2 hours in gelatine-coated dishes. On day 1 , the medium was changed to human embryoid body (hEB) medium containing DMEM/F-12, 5\% KSR, 2 mM Lglutamine, 1\% MEM Non-Essential Amino Acids (NEAA), and $0.1 \mathrm{mM} 2-$ mercaptoethanol with 300 nM LDN-193189 (Sigma-Aldrich, USA), 3 M SB431542 (Tocris, UK), and $3 \mu \mathrm{M} \mathrm{CHIR99021} \mathrm{(Focus} \mathrm{Biomolecules,} \mathrm{USA).} \mathrm{On} \mathrm{day} \mathrm{2,} \mathrm{the} \mathrm{medium}$ was changed to fresh hEB medium containing 300 nM LDN-193189, 3 M SB431542, $3 \mu \mathrm{M}$ CHIR99021, and $1 \mu \mathrm{M}$ retinoic acid (RA) (Sigma-Aldrich, USA). From day 4 to 
day 14 , hEBs were cultured in hEB medium containing $1 \mu \mathrm{M} \mathrm{RA}$ and $1 \mu \mathrm{M}$ purmorphamine (Calbiochem, Germany), and the medium was changed every 2-3 days. On day 14, hEBs were enzymatically dissociated into single cells using TrypLE Select (Thermo Fisher Scientific, USA). The dissociated cells were plated on growth factor-reduced Matrigel (33 × dilution, thin coating; Corning, USA)-coated dishes at a density of $2 \times 10^{4}$ cells/well (96-well imaging plate, Greiner Bio-One, Austria) and cultured in MN medium (MNM) consisting of KBM Neural Stem Cell medium (Kohjin Bio, Japan) supplemented with 2\% B27 supplement (Thermo Fisher Scientific, USA), 1\% NEAA, 50 nM RA, 500 nM purmorphamine, $10 \mu$ M cyclic AMP (cAMP) (SigmaAldrich, USA), $10 \mathrm{ng} / \mathrm{mL}$ recombinant BDNF (R\&D Systems, USA), $10 \mathrm{ng} / \mathrm{mL}$ recombinant GDNF (R\&D Systems, USA), $10 \mathrm{ng} / \mathrm{mL}$ recombinant human IGF-1 (R\&D Systems, USA), and $200 \mathrm{ng} / \mathrm{mL}$ L-ascorbic acid (Sigma-Aldrich, USA) for up to 4 weeks. Half of the medium was changed every 2-3 days. To visualize HB9-positive MNs, cells were infected with $H B 9^{e 438}:$ Venus lentivirus on day 4 as previously described ${ }^{33}$. All the experimental procedures for the production and the use of iPSCs were approved by the ethics committee of the Aichi Medical University School of Medicine (approval number 14-004 and 2020-213).

\section{Immunocytochemical staining}

Cells were fixed in 4\% paraformaldehyde for 15-25 min at room temperature and permeabilized with $0.1 \%$ Triton-X. Nonspecific binding was blocked by incubation with $1 \%$ normal goat serum with $0.05 \%$ Triton $\mathrm{X}-100$ for HeLa cells or $10 \%$ normal goat serum with $0.3 \%$ Triton $\mathrm{X}-100$ for iPSC-derived MNs. Cells were then incubated with primary antibodies overnight at $4{ }^{\circ} \mathrm{C}$ in PBS containing $1 \% \mathrm{BSA}$ and $0.05 \%$ Triton $\mathrm{X}$ 100 , rinsed with PBS three times, and incubated with Alexa Fluor-conjugated secondary antibodies (Thermo Fisher Scientific, USA) for 2 hours at room temperature. Nuclei were stained with $10 \mu \mathrm{g} / \mathrm{ml}$ Hoechst 33258 (Sigma-Aldrich, USA). The cells were then rinsed with PBS three times. The following antibodies were used: mouse anti-FLAG (F3165, Sigma, 1:200), rabbit anti-FLAG (F7425, Sigma, 1:200), rat antiFLAG (NBP1-06712, Novus, 1:50), rabbit anti-PABP (ab21060, Abcam, 1:1000), 
mouse anti-PABP (P6246, Sigma, 1:500), mouse anti-SMN (IgG1, 610646, BD Biosciences, 1:250), mouse anti-Gemin2 (IgG2b, sc-32806, Santa Cruz, 1:50), goat anti-GFP (600-101-215, ROCKLAND, 1:500), rabbit anti-GFP (598, MBL, 1:500), mouse anti-HB9 (IgG1, 81.5C10, Developmental Studies Hybridoma Bank, 1:1000), mouse anti-Isl-1 (IgG2b, 39.4D5, Developmental Studies Hybridoma Bank, 1:400), and mouse anti- $\beta$ III-tubulin (IgG2a, MMS-435P, Covance, 1:2000). Alexa Fluor 488-, Alexa Fluor 555-, Alexa Fluor 594-, or Alexa Fluor 647-conjugated anti-mouse, anti-rabbit, anti-mouse lgG1, anti-mouse $\lg G 2 a$, and anti-mouse lgG2b antibodies were used. HeLa cells were mounted using Dako Fluorescence Mounting Medium (S3023, Agilent Technology, USA).

Images of MNs differentiated from iPSCs and HeLa cells were obtained using a confocal microscope LSM700 (Carl Zeiss Microscopy, Germany) and a Keyence BZX700 microscope (Keyence, Japan), respectively. Images were analysed using Adobe Photoshop. For the counting of GEM in MNs differentiated from iPSCs, all Z-stack images at $2 \mu \mathrm{m}$ intervals were analysed. SMN-positive granules in nuclei were counted as GEM in HeLa cells. SMN- and Gemin2-positive granules in nuclei were counted as GEM in MNs differentiated from iPSCs.

\section{RNA staining}

Cells were prewashed with PBS and incubated with 500 mM SYTO RNASelect Green Fluorescent Cell Stain (S32703, Thermo Fisher Scientific, USA) for 20 min at $37^{\circ} \mathrm{C}$, according to the instructions. Cells were then rinsed in medium and fixed with methanol for $10 \mathrm{~min}$ at $-20^{\circ} \mathrm{C}$, followed by immunostaining.

\section{Statistical analysis}

Statistical analysis was performed using Prism 9 (GraphPad software, USA). Statistical tests included one-way and two-way ANOVA with Tukey's multiple comparisons tests. All experiments are represented as the average \pm standard error of the mean. 


\section{References}

1 Taylor, J. P., Brown, R. H., Jr. \& Cleveland, D. W. Decoding ALS: from genes to mechanism. Nature 539, 197-206, doi:10.1038/nature20413 (2016).

2 Renton, A. E. et al. A hexanucleotide repeat expansion in C9ORF72 is the cause of chromosome 9p21-linked ALS-FTD. Neuron 72, 257-268, doi:10.1016/j.neuron.2011.09.010 (2011).

3 DeJesus-Hernandez, M. et al. Expanded GGGGCC hexanucleotide repeat in noncoding region of C9ORF72 causes chromosome 9p-linked FTD and ALS. Neuron 72, 245-256, doi:10.1016/j.neuron.2011.09.011 (2011).

4 Ash, P. E. et al. Unconventional translation of C9ORF72 GGGGCC expansion generates insoluble polypeptides specific to c9FTD/ALS. Neuron 77, 639-646, doi:10.1016/j.neuron.2013.02.004 (2013).

$5 \mathrm{Zu}, \mathrm{T}$. et al. RAN proteins and RNA foci from antisense transcripts in C9ORF72 ALS and frontotemporal dementia. Proc Natl Acad Sci U S A 110, E4968-4977, doi:10.1073/pnas.1315438110 (2013).

6 Mori, K. et al. The C9orf72 GGGGCC repeat is translated into aggregating dipeptide-repeat proteins in FTLD/ALS. Science 339, 1335-1338, doi:10.1126/science.1232927 (2013).

$7 \quad$ Kwon, I. et al. Poly-dipeptides encoded by the C9orf72 repeats bind nucleoli, impede RNA biogenesis, and kill cells. Science 345, 1139-1145, doi:10.1126/science.1254917 (2014).

8 Mizielinska, S. et al. C9orf72 repeat expansions cause neurodegeneration in Drosophila through arginine-rich proteins. Science 345, 1192-1194, doi:10.1126/science.1256800 (2014).

$9 \quad$ Wen, X. et al. Antisense proline-arginine RAN dipeptides linked to C9ORF72ALS/FTD form toxic nuclear aggregates that initiate in vitro and in vivo neuronal death. Neuron 84, 1213-1225, doi:10.1016/j.neuron.2014.12.010 (2014).

10 Gitler, A. D. \& Tsuiji, H. There has been an awakening: Emerging mechanisms of C9orf72 mutations in FTD/ALS. Brain Res 1647, 19-29, doi:10.1016/j.brainres.2016.04.004 (2016).

11 Saberi, S. et al. Sense-encoded poly-GR dipeptide repeat proteins correlate to neurodegeneration and uniquely co-localize with TDP-43 in dendrites of repeat- 
expanded C9orf72 amyotrophic lateral sclerosis. Acta Neuropathol 135, 459474, doi:10.1007/s00401-017-1793-8 (2018).

12 Sakae, N. et al. Poly-GR dipeptide repeat polymers correlate with neurodegeneration and Clinicopathological subtypes in C9ORF72-related brain disease. Acta Neuropathol Commun 6, 63, doi:10.1186/s40478-018-0564-7 (2018).

13 Lefebvre, S. et al. Identification and characterization of a spinal muscular atrophy-determining gene. Cell 80, 155-165, doi:10.1016/0092-8674(95)904603 (1995).

14 Burghes, A. H. \& Beattie, C. E. Spinal muscular atrophy: why do low levels of survival motor neuron protein make motor neurons sick? Nat Rev Neurosci 10, 597-609, doi:10.1038/nrn2670 (2009).

15 Coady, T. H. \& Lorson, C. L. SMN in spinal muscular atrophy and snRNP biogenesis. Wiley Interdiscip Rev RNA 2, 546-564, doi:10.1002/wrna.76 (2011).

16 Tsuiji, H. \& Yamanaka, K. Animal Models for Neurodegerative Disorders. 3956 (Elsevier Inc., 2014) doi: 10.1016/B978-0-12-416002-6.00003-1.

17 Wan, L. et al. The survival of motor neurons protein determines the capacity for snRNP assembly: biochemical deficiency in spinal muscular atrophy. Mol Cell Biol 25, 5543-5551, doi:10.1128/MCB.25.13.5543-5551.2005 (2005).

18 Zhang, Z. et al. SMN deficiency causes tissue-specific perturbations in the repertoire of snRNAs and widespread defects in splicing. Cell 133, 585-600, doi:10.1016/j.cell.2008.03.031 (2008).

19 Lefebvre, S. et al. Correlation between severity and SMN protein level in spinal muscular atrophy. Nat Genet 16, 265-269, doi:10.1038/ng0797-265 (1997).

20 Matera, A. G., Terns, R. M. \& Terns, M. P. Non-coding RNAs: lessons from the small nuclear and small nucleolar RNAs. Nat Rev Mol Cell Biol 8, 209-220, doi:10.1038/nrm2124 (2007).

21 Patel, A. A. \& Steitz, J. A. Splicing double: insights from the second spliceosome. Nat Rev Mol Cell Biol 4, 960-970, doi:10.1038/nrm1259 (2003).

22 Tsuiji, H. et al. Spliceosome integrity is defective in the motor neuron diseases ALS and SMA. EMBO Mol Med 5, 221-234, doi:10.1002/emmm.201202303 (2013).

23 Ishihara, T. et al. Decreased number of Gemini of coiled bodies and U12 snRNA 
level in amyotrophic lateral sclerosis. Hum Mol Genet 22, 4136-4147, doi:10.1093/hmg/ddt262 (2013).

24 Yamazaki, T. et al. FUS-SMN protein interactions link the motor neuron diseases ALS and SMA. Cell Rep 2, 799-806, doi:10.1016/j.celrep.2012.08.025 (2012).

25 Shan, X., Chiang, P. M., Price, D. L. \& Wong, P. C. Altered distributions of Gemini of coiled bodies and mitochondria in motor neurons of TDP-43 transgenic mice. Proc Natl Acad Sci U S A 107, 16325-16330, doi:10.1073/pnas.1003459107 (2010).

26 Kariya, S. et al. Mutant superoxide dismutase 1 (SOD1), a cause of amyotrophic lateral sclerosis, disrupts the recruitment of SMN, the spinal muscular atrophy protein to nuclear Cajal bodies. Hum Mol Genet 21, 34213434, doi:10.1093/hmg/dds174 (2012).

27 Perera, N. D. et al. Enhancing survival motor neuron expression extends lifespan and attenuates neurodegeneration in mutant TDP-43 mice. Hum Mol Genet 25, 4080-4093, doi:10.1093/hmg/ddw247 (2016).

28 Turner, B. J. et al. Overexpression of survival motor neuron improves neuromuscular function and motor neuron survival in mutant SOD1 mice. Neurobiol Aging 35, 906-915, doi:10.1016/j.neurobiolaging.2013.09.030 (2014).

29 Sun, S. et al. ALS-causative mutations in FUS/TLS confer gain and loss of function by altered association with SMN and U1-snRNP. Nat Commun 6, 6171, doi:10.1038/ncomms7171 (2015).

30 Wan, L., Ottinger, E., Cho, S. \& Dreyfuss, G. Inactivation of the SMN complex by oxidative stress. Mol Cell 31, 244-254, doi:10.1016/j.molcel.2008.06.004 (2008).

31 Lee, K. H. et al. C9orf72 Dipeptide Repeats Impair the Assembly, Dynamics, and Function of Membrane-Less Organelles. Cell 167, 774-788 e717, doi:10.1016/j.cell.2016.10.002 (2016).

32 Boeynaems, S. et al. Phase Separation of C9orf72 Dipeptide Repeats Perturbs Stress Granule Dynamics. Mol Cell 65, 1044-1055 e1045, doi:10.1016/j.molcel.2017.02.013 (2017).

33 Shimojo, D. et al. Rapid, efficient, and simple motor neuron differentiation from human pluripotent stem cells. Mol Brain 8, 79, doi:10.1186/s13041-015-0172-4 
(2015).

34 Onodera, K. et al. Unveiling synapse pathology in spinal bulbar muscular atrophy by genome-wide transcriptome analysis of purified motor neurons derived from disease specific iPSCs. Mol Brain 13, 18, doi:10.1186/s13041020-0561-1 (2020).

35 Okada, R. et al. Modulation of oxygen tension, acidosis, and cell density is crucial for neural differentiation of human induced pluripotent stem cells. Neurosci Res 163, 34-42, doi:10.1016/j.neures.2020.01.015 (2021).

36 Zhang, K. et al. The C9orf72 repeat expansion disrupts nucleocytoplasmic transport. Nature 525, 56-61, doi:10.1038/nature14973 (2015).

37 Freibaum, B. D. et al. GGGGCC repeat expansion in C9orf72 compromises nucleocytoplasmic transport. Nature 525, 129-133, doi:10.1038/nature14974 (2015).

38 Jovicic, A. et al. Modifiers of C9orf72 dipeptide repeat toxicity connect nucleocytoplasmic transport defects to FTD/ALS. Nat Neurosci 18, 1226-1229, doi:10.1038/nn.4085 (2015).

39 Boeynaems, S. et al. Drosophila screen connects nuclear transport genes to DPR pathology in c9ALS/FTD. Sci Rep 6, 20877, doi:10.1038/srep20877 (2016).

40 Zhang, Y. J. et al. Poly(GR) impairs protein translation and stress granule dynamics in C9orf72-associated frontotemporal dementia and amyotrophic lateral sclerosis. Nat Med 24, 1136-1142, doi:10.1038/s41591-018-0071-1 (2018).

41 Zhang, Y. J. et al. Heterochromatin anomalies and double-stranded RNA accumulation underlie C9orf72 poly(PR) toxicity. Science 363, doi:10.1126/science.aav2606 (2019).

42 Lin, Y. et al. Toxic PR Poly-Dipeptides Encoded by the C9orf72 Repeat Expansion Target LC Domain Polymers. Cell 167, 789-802 e712, doi:10.1016/j.cell.2016.10.003 (2016).

43 Lopez-Gonzalez, R. et al. Poly(GR) in C9ORF72-Related ALS/FTD Compromises Mitochondrial Function and Increases Oxidative Stress and DNA Damage in iPSC-Derived Motor Neurons. Neuron 92, 383-391, doi:10.1016/j.neuron.2016.09.015 (2016). 
44 Yin, S. et al. Evidence that C9ORF72 Dipeptide Repeat Proteins Associate with U2 snRNP to Cause Mis-splicing in ALS/FTD Patients. Cell Rep 19, 2244-2256, doi:10.1016/j.celrep.2017.05.056 (2017).

45 Bernabo, P. et al. In Vivo Translatome Profiling in Spinal Muscular Atrophy Reveals a Role for SMN Protein in Ribosome Biology. Cell Rep 21, 953-965, doi:10.1016/j.celrep.2017.10.010 (2017).

46 Lauria, F. et al. SMN-primed ribosomes modulate the translation of transcripts related to spinal muscular atrophy. Nat Cell Biol 22, 1239-1251, doi:10.1038/s41556-020-00577-7 (2020).

47 Rodriguez-Muela, N. et al. Single-Cell Analysis of SMN Reveals Its Broader Role in Neuromuscular Disease. Cell Rep 18, 1484-1498, doi:10.1016/j.celrep.2017.01.035 (2017).

48 Takahashi, K., Okita, K., Nakagawa, M. \& Yamanaka, S. Induction of pluripotent stem cells from fibroblast cultures. Nat Protoc 2, 3081-3089, doi:10.1038/nprot.2007.418 (2007).

49 Imamura, K. et al. The Src/c-Abl pathway is a potential therapeutic target in amyotrophic lateral sclerosis. Sci Transl Med 9, doi:10.1126/scitransImed.aaf3962 (2017).

\section{Acknowledgements}

This work was supported by Japan Society for the Promotion of Science KAKENHI Grant Numbers JP17K08280 and JP20K07016; the ALS Foundation, Japan ALS Association; the Ichihara International Scholarship Foundation; Grant-in-Aid for Research in Nagoya City University Grant Numbers 1 and 2022004 to HT. This work was also supported by grants from the Practical Research Project for Intractable Diseases and Research Center Network for Realization of Regenerative Medicine of the Japan Agency for Medical Research and Development (AMED) (JP19ek0109243 and JP20bm0804020) to YO. 


\section{Author contributions}

M.Y., I.N., and H.T. investigated and prepared figures 1-3. Y.K., I.N., K.O., Y.O., and H.T. investigated and prepared figures 4. Y.O. and H.T. wrote the main manuscript text. H.O., H.I., M.H., and Y.O. provided resources. All authors reviewed the manuscript.

\section{Conflict of interest}

Y.O. is a scientific advisor of Kohjin Bio Co. Ltd., Japan. H.O. is a founding scientist of SanBio Co. Ltd., Japan and K Pharma Inc., Japan. The other authors declare that they have no competing interests.

\section{Figure Legends}

\section{Figure 1. C9ORF72 poly(GR) disrupts GEM formation}

(a) HeLa-C3 cells were transfected with empty vector (mock), a FLAG-tagged construct expressing 50 repeats of GR (poly $(G R)$ ) or a FLAG-tagged construct expressing 50 repeats of PR (poly(PR)) and immunostained with anti-FLAG and anti-SMN antibodies. SMN-positive nuclear granules are GEMs. Nuclei were counterstained with Hoechst. Scale bar $=10 \mu \mathrm{m}$.

(b) Quantification of the number of GEMs visualized in (A). Cells transfected with empty vector (mock), cells with an accumulation of poly(GR) in the nucleolus, and cells with poly(PR) were analysed. More than 100 cells were counted. Biological replicates; $n=3 \sim 5$. One-way ANOVA with Tukey's multiple comparisons tests, ${ }^{* * * * ;}$ $\mathrm{p}<0.0001$.

Figure 2. C9ORF72 poly(GR) and poly(PR) induce cytoplasmic RNA granules

\section{harboring SMN protein}

(a) HeLa-C3 cells were transfected with empty vector (mock), FLAG-poly(GR), or FLAG-poly(PR) and immunostained with anti-FLAG and anti-PABP antibodies. 
PABP was distributed diffusely in the cytoplasm of cells with mock transfection, whereas PABP formed foci in the cytoplasm of cells with poly(GR) or poly(PR). Notably, PABP-granules contained poly(GR) but not poly(PR). Nuclei were counterstained with Hoechst. Cytoplasmic granules are indicated with arrowheads. Scale bar $=10 \mu \mathrm{m}$.

(b) HeLa-C3 cells were transfected with empty vector (mock), FLAG-poly(GR), or FLAG-poly(PR) and immunostained with anti-FLAG and anti-SMN antibodies. SMN formed foci in the cytoplasm of cells with poly(GR) or poly(PR). SMN occasionally colocalized with poly(GR) in the cytoplasm. Nuclei were counterstained with Hoechst. Cytoplasmic granules are indicated with arrowheads. Scale bar $=10 \mu \mathrm{m}$.

(c) HeLa-C3 cells were transfected with empty vector (mock), FLAG-poly(GR), or FLAG-poly(PR) and immunostained with anti-SMN and anti-PABP antibodies. SMN occasionally colocalized with PABP. Nuclei were counterstained with Hoechst. Cytoplasmic granules are indicated with arrowheads. Scale bar $=10 \mu \mathrm{m}$.

(d) HeLa-C3 cells were transfected with empty vector (mock) or FLAG-poly(GR), and RNA was visualized with SYTO RNAselect (Molecular Probes). Nuclei were counterstained with Hoechst. Cytoplasmic granules are indicated with arrowheads. Scale bar $=10 \mu \mathrm{m}$.

(e) Quantification of the number of cytoplasmic PABP granules visualized in (a). Cells transfected with empty vector (mock), cells with an accumulation of poly(GR) in the nucleolus, and cells with poly(PR) were analysed. More than 100 cells were counted. Biological replicates; $n=3 \sim 5$. One-way ANOVA with Tukey's multiple comparisons tests, ${ }^{*} ; p<0.05,{ }^{* * *} ; p<0.0001$.

\section{Figure 3. Persistent cytoplasmic stress granules induced by poly(PR) and} oxidative stress sequester SMN

(a) Quantification of the number of cytoplasmic stress granules induced by poly(PR) and oxidative stress. HeLa-C3 cells were treated with $500 \mu \mathrm{M}$ sodium arsenite for 
$30 \mathrm{~min}$ to induce cytoplasmic stress granules. Cells were then washed to remove the arsenite and allowed to recover and the stress granules to disassemble. Cells were fixed at the indicated time points and immunostained with anti-FLAG and antiPABP antibodies. Cells with cytoplasmic PABP granules were counted. More than 100 cells were counted. Biological replicates; $n=3 \sim 5$. Two-way ANOVA with Tukey's multiple comparisons tests, ${ }^{* * *} ; p<0.0001$.

(b) Immunostained images of persistent cytoplasmic granules induced in (a). Cells were immunostained with anti-FLAG, anti-PABP, and anti-SMN antibodies. Scale bar $=10 \mu \mathrm{m}$. Nuclei counterstained with Hoechst are enclosed by dotted lines.

\section{Figure 4. The number of GEMs in MNs derived from iPSCs of a C9ORF72-ALS}

\section{patient is reduced}

(a) Schematic of the protocol for the induction of MNs from iPSCs of healthy subjects and a C9ORF72-ALS patient ${ }^{33}$. LSC: LDN-193189, SB431542, and CHIR99021, RA: retinoic acid.

(b) Differentiation of MNs from iPSCs. Cells were fixed and analysed with anti-IsI-1, anti-HB9, and anti- $\beta$ III tubulin antibodies on day 7 of adherent differentiation. The differentiation efficiencies for the three iPSCs were approximately the same. Nuclei were counterstained with Hoechst. Scale bar $=20 \mu \mathrm{m}$.

(c) Quantification of the number of GEMs in MNs differentiated from iPSCs of healthy donors and a C9ORF72-ALS patient. MNs were stained with anti-GFP (HB9 ${ }^{e 436 .: V e n u s), ~ a n t i-S M N, ~ a n d ~ a n t i-G e m i n ~} 2$ antibodies at 4 weeks of differentiation. MNs were identified by HB9 ${ }^{\mathrm{e} 366}:$ :Venus expression. SMN- and Gemin 2-double positive granules were counted as GEMs. More than 100 cells were counted. Biological replicates; $n=3$. One-way ANOVA with Tukey's multiple comparisons tests, ${ }^{* * *} ; p<0.001$.

(d) Representative confocal images of GEMs in MNs differentiated from iPSCs of healthy donors and a C9ORF72-ALS patient at 4 weeks of adherent differentiation. 
SMN- and Gemin 2-double positive granules were counted as GEMs. Arrows indicate GEMs. Scale bar $=10 \mu \mathrm{m}$.

Figure 5. Graphical representation of the overall findings of this study In normal cells, SMN protein forms phase-separated foci called GEMs in the nucleus and is diffusely distributed in the cytoplasm. Accumulation of C9ORF72 poly(GR) and poly(PR) disturbs GEM formation and induces cytoplasmic granules harboring SMN and RNA-binding proteins. Poly(PR) expression combined with oxidative stress induces persistent cytoplasmic stress granules harboring SMN and RNA-binding proteins.

\section{Table}

\begin{tabular}{|c|c|c|c|c|c|c|c|c|}
\hline & $\begin{array}{l}\text { clone name } \\
\text { at } \\
\text { establishment }\end{array}$ & gender & $\begin{array}{l}\text { onset } \\
\text { age } \\
\text { (y) }\end{array}$ & $\begin{array}{l}\text { age at } \\
\text { diagnosis }\end{array}$ & $\begin{array}{l}\text { biopsy } \\
\text { age } \\
\text { (y) }\end{array}$ & origin & reprogramming & references \\
\hline Control 1 & TIGE-9 & male & N.A. & N.A. & 36 & skin fibroblasts & episomal & Shimojo et al., $2015^{33}$ \\
\hline Control 2 & 201B7 & female & N.A. & N.A. & 36 & skin fibroblasts & retrovirus & Takahashi et al., 200748 \\
\hline C9-ALS & ND06769E4 & female & 45 & 46 & 46 & $\begin{array}{l}\text { immortalized } \\
\text { B-lymphocytes }\end{array}$ & episomal & Imamura et al., $2017^{49}$ \\
\hline
\end{tabular}

\section{Table 1. List of iPSC clones}


bioRxiv preprint doi: https://doi.org/10.1101/2021.03.24.436890; this version posted March 25, 2021. The copyright holder for this preprint

Kato et al. Figure 1

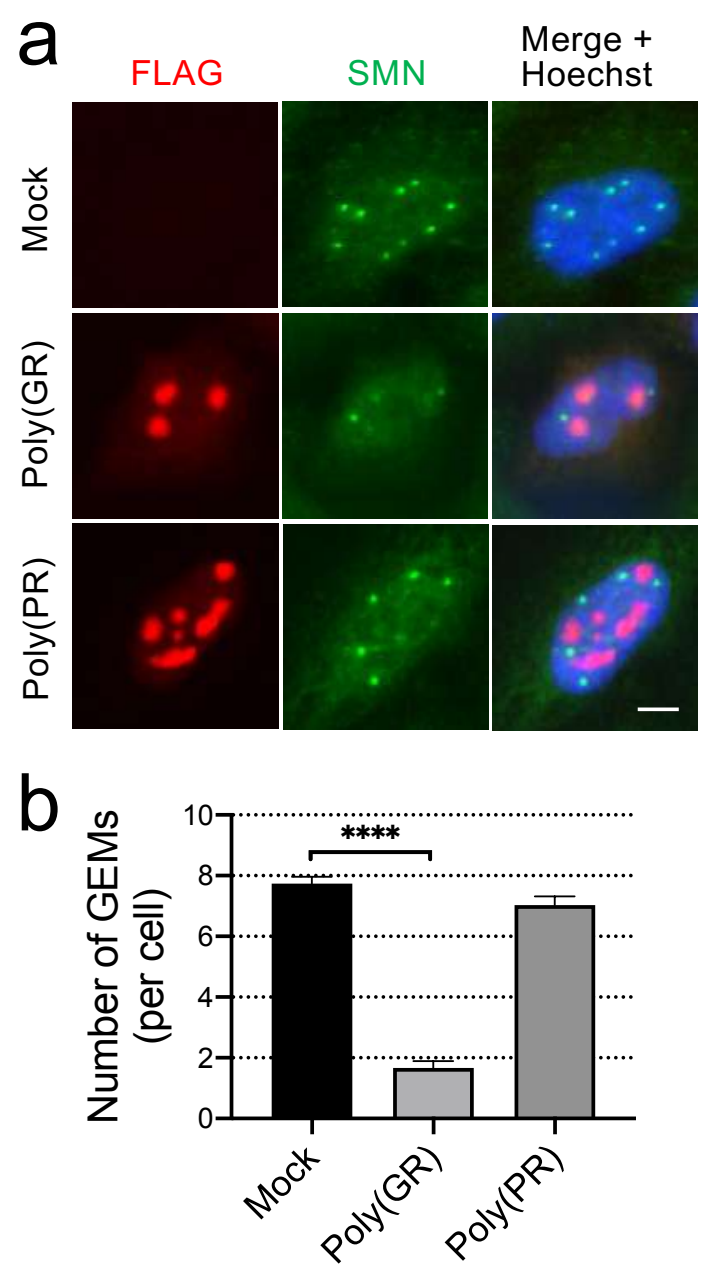




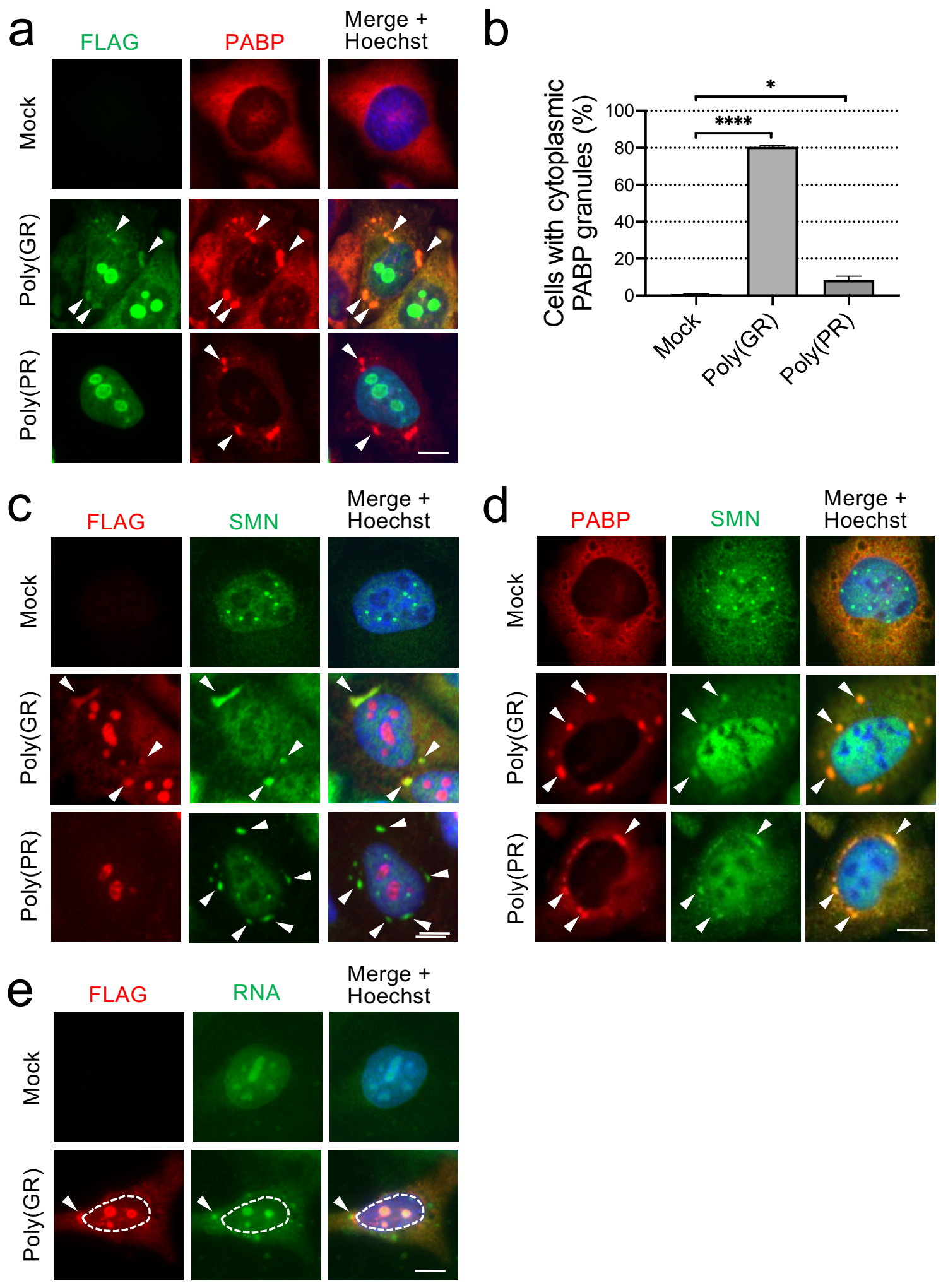


Kato et al. Figure 3
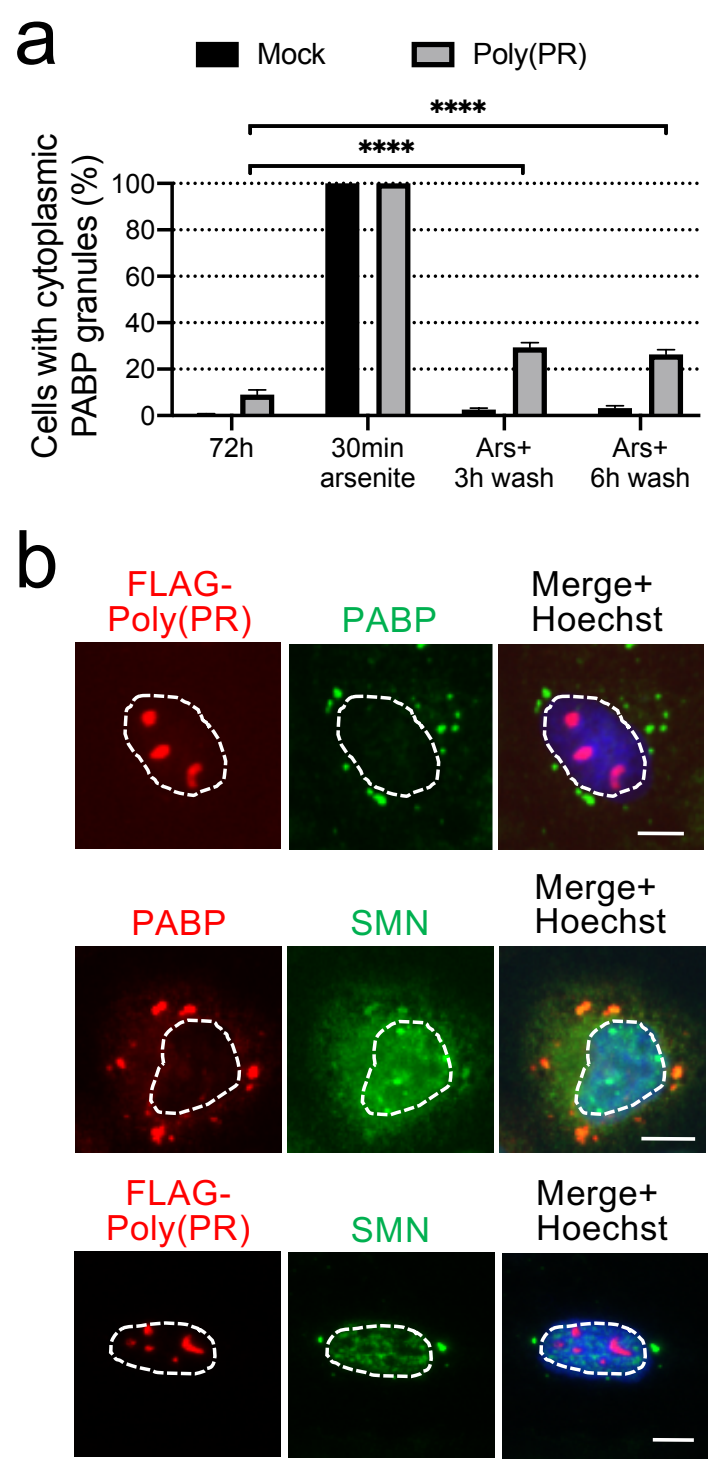


\section{Kato et al. Figure 4}

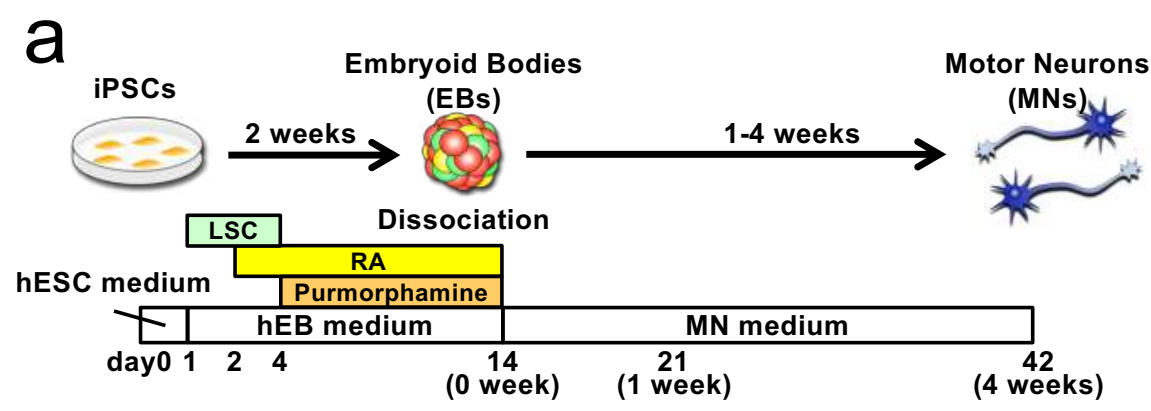

b

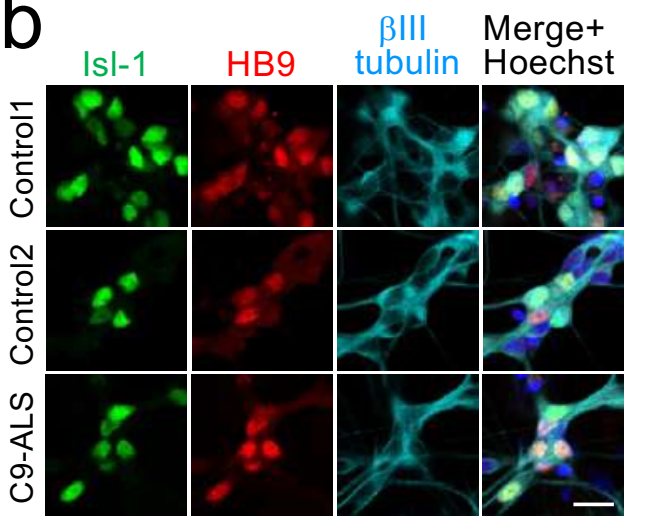

C
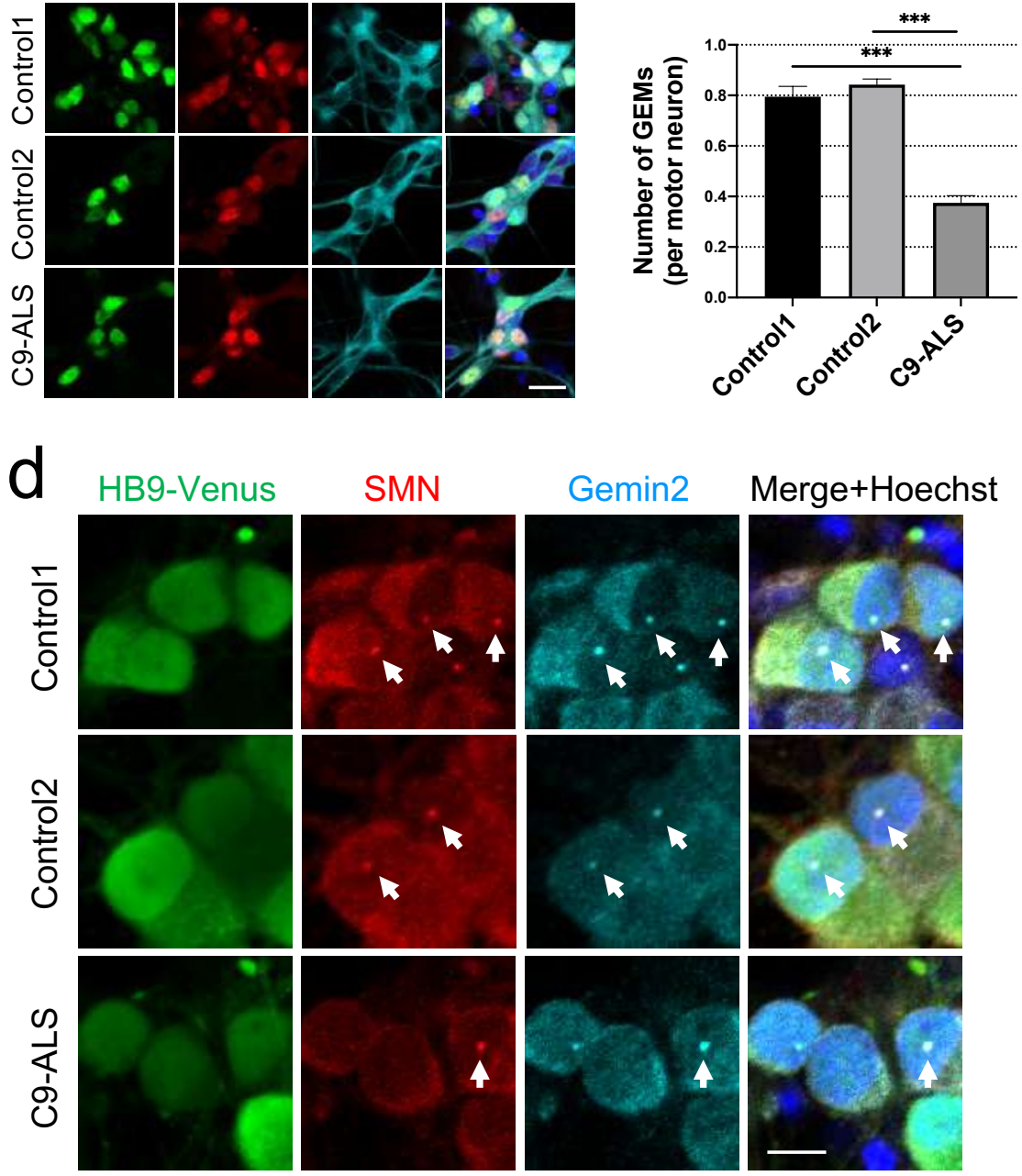
Loss of GEMs

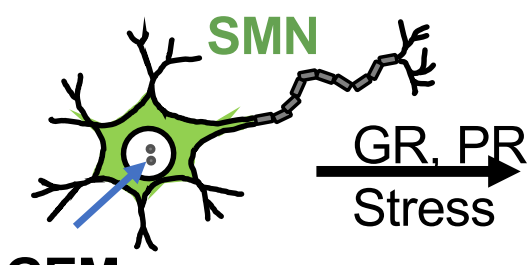

GEMs

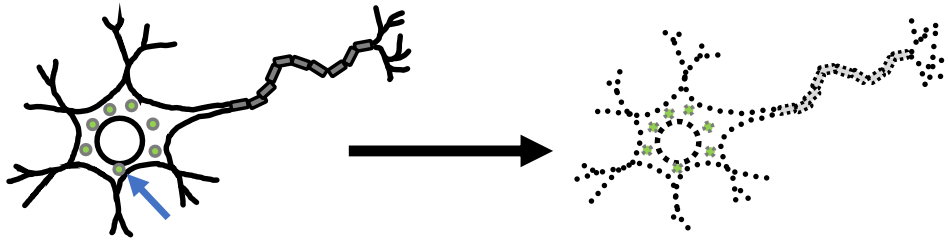

SMN granules in cytoplasm
Neurodegeneration $\begin{aligned} \text { DPRs } & \rightarrow \text { Loss of GEM } \\ & \text { SMN granules in cytoplasm }\end{aligned}$

DPRs \& oxidative stress $\rightarrow$ Loss of GEM 